\title{
Formation of $\boldsymbol{N}$-Acetylputrescine and $\boldsymbol{N}^{1}$-Acetylspermidine in Cultured Human Lymphocytes
}

\author{
Moshe MENASHE, ${ }^{*}$ Joseph FABER* and Uriel BACHRACH† \\ *Shaare Zedek Medical Center, Jerusalem, Israel, and †Department of Molecular Biology, The Hebrew \\ University-Hadassah Medical School, Jerusalem, Israel
}

(Received 20 August 1979)

\begin{abstract}
$N$-Acetylputrescine and $N^{1}$-acetylspermidine were synthesized from putrescine in cultured human lymphocytes. They were identified by paper chromatography and t.l.c. Acetylputrescine was also identified by mass spectrometry. $N$-Acetylputrescine and $N^{1}$-acetylspermidine were formed in untreated cells and in lectin-transformed cells.
\end{abstract}

The naturally occurring polyamines, putrescine, spermidine and spermine, have been implicated in many growth processes and in differentiation (Cohen, 1971; Bachrach, 1973; Russell \& Durie, 1978). Their concentrations and the activity of their biosynthetic enzymes increase significantly in proliferating cells (Tabor \& Tabor, 1976; Jänne \& Raina, 1968) and neoplastic tissues (Bachrach, 1978; Russell \& Durie, 1978). One of the models investigated is the lymphocyte induced to blastogenesis by a lectin. It has been shown that cellular polyamine concentrations increase significantly during the transformation process (Morris, 1978), as do the biosynthetic decarboxylases, i.e. ornithine decarboxylase and $S$-adenosyl-L-methionine decarboxylase (Fillingame \& Morris, 1973; Kay \& Lindsay, 1973).

Several polyamine-catabolic pathways are reported in the literature. Oxidation of polyamines has been studied extensively and $\gamma$-aminobutyrate has been isolated as the oxidation product of putrescine (Bachrach, 1973). In addition, there are several lines of evidence suggesting that polyamines undergo acetylation. The mono-acetyl derivative of the diamine putrescine was found in various tissues, including human brain (Perry et al., 1967), and in several organs of other vertebrates (Seiler et al., 1973). $N^{1}$ - and $N^{8}$-Acetylspermidine have been shown to occur in the urine of normal subjects and cancer patients (Abdel-Monem et al., 1975).

In the present study we used human lymphocytes, stimulated for transformation, as a model for normal human proliferating cells, and we present evidence that $N$-acetylputrescine and $N^{1}$-acetylspermidine are formed in these cells when they are grown in vitro in the presence of $\left[{ }^{14} \mathrm{C}\right]$ putrescine.

‡ To whom reprint requests should be addressed.
The acetylated polyamine derivatives are subsequently released into the growth medium.

\section{Materials and Methods}

\section{Materials}

Blood samples were obtained from healthy human volunteers post-prandially. The blood was drawn with heparin as an anticoagulant. After separation of the plasma, cells were mixed with medium RPMI 1640 (Bio-Lab Laboratories, Jerusalem, Israel), and mononuclear cells were separated at room temperature by using an Isolymph (Gallard-Schlesinger Chemical Corp., New York, NY, U.S.A.) gradient as described elsewhere (Thorsby \& Bratlie, 1970). The lymphocytes $\left(2 \times 10^{6}\right.$ cells $\left./ \mathrm{ml}\right)$ were cultured in sterile plastic tubes (NUNC, Roskilde, Denmark), in a humidified atmosphere of $5 \% \mathrm{CO}_{2}$ in air, at $37^{\circ} \mathrm{C}$. The culture medium consisted of $5 \mathrm{ml}$ of RPMI 1640 medium supplemented with combined antibiotics: penicillin $200 \mathrm{mg}$, streptomycin $200 \mathrm{mg}$, neomycin $10 \mathrm{mg}$, kanamycin $10 \mathrm{mg}$ and $2 \mathrm{mmol}$ of L-glutamine per litre of medium.

Autologous plasma was added to a final concentration of $10 \%(\mathrm{v} / \mathrm{v})$. Purified phytohaemagglutinin (Wellcome, Beckenham, Kent, U.K.) at a concentration of $1 \mu \mathrm{g} / \mathrm{ml}$ served as a mitogen. $\left[1,4-{ }^{14} \mathrm{C}\right]$ Putrescine $(1.0 \mu \mathrm{Ci} /$ culture; sp. radioactivity $89.9 \mathrm{mCi} / \mathrm{mmol}$; New England Nuclear Corp., Boston, MA, U.S.A.) was added to the culture at zero time. Transformation was estimated by $\left[{ }^{3} \mathrm{H}\right]$ thymidine incorporation $(2 \mu \mathrm{Ci} / \mathrm{ml}$; sp. radioactivity $2 \mathrm{Ci} / \mathrm{mmol}$; Nuclear Research Center, Negev, Israel) after incubation for $3 \mathrm{~h}$. For assay cells were sedimented by centrifugation $(750 \mathrm{~g}$ for $10 \mathrm{~min})$ and the growth medium was removed and freezedried. The pellet was washed in $0.9 \%(w / v) ~ \mathrm{NaCl}$ 
and centrifuged again. Polyamines were extracted with 3\% (v/v) $\mathrm{HClO}_{4}$, from both cells and the freeze-dried medium.

\section{Methods}

Polyamine separation. Polyamines were separated by paper chromatography on Whatman no. 3 paper by using different solvents: a, propan-2-ol/85\% (v/v) formic acid/water (8:1:1, by vol.) (Noto et al., 1978; Kakimoto et al., 1969); b, propan1-ol/triethylamine/water $(85: 3: 15$, by vol.) (Dubin \& Rosenthal, 1960). Dansyl (5-dimethylaminonaphthalene-1-sulphonyl) derivatives were prepared as described by Seiler \& Wiechmann (1967), and separated on silica-gel plates (Kodak 13179 Eastman Chromatogram Sheet, $100 \mu \mathrm{m}$ thick), by using several solvents: benzene/methanol $(19: 1, \mathrm{v} / \mathrm{v})$, cyclohexane/ethyl acetate $(3: 2, \mathrm{v} / \mathrm{v})$, chloroform/propan-2-ol $(10: 1, \mathrm{v} / \mathrm{v})$. The plates were apposed to films (Kodak X-ray Noscreen) for several days. Radioactive material identified by this radioautography was scraped off the plates and extracted with $1-2 \mathrm{ml}$ of ethyl acetate for quantification and for further identification. As markers we used $\left[{ }^{14} \mathrm{C}\right]$ spermidine and $\left[{ }^{14} \mathrm{C}\right]$ spermine (New England Nuclear Corp.). $\left[{ }^{3} \mathrm{H}\right]$ Acetylputrescine and $N^{1}$ - and $N^{8}$-acetylspermidine were kindly given by Dr. N. Seiler (Centre de Recherche Merrell International, Strasbourg, France).

Fluorescent dansyl derivatives were extracted from distinct spots of the thin-layer chromatograms and analysed in a L. K. B. mass spectrometer (Seiler et al., 1970).

\section{Results}

Fig. 1 shows that the formation of radioactive spermidine and spermine from $\left[{ }^{14} \mathrm{C}\right]$ putrescine takes place in the transformed cells, but hardly in the unstimulated ones. Two radioactive compounds, which were later identified as acetyl derivatives of putrescine and spermidine, occurred in the growth medium of the transformed and unstimulated lymphocytes. These compounds were not detected when growth medium was incubated with plasma without cells. Therefore it is most likely that they are formed within the cells. Fig. 2 shows that acetylputrescine is readily released into the medium and $\mathrm{N}$-acetylspermidine is released to a lesser extent to the medium and tends to accumulate within the cells. Formation of spermidine and spermine by lymphocytes has been documented (see Morris, 1978), but the occurrence of acetylated polyamine derivatives has not yet been reported. Therefore it was desirable to identify these products and to establish their chemical nature. Samples of medium were separated by paper chromatography.

Table 1 shows the occurrence of two compounds:

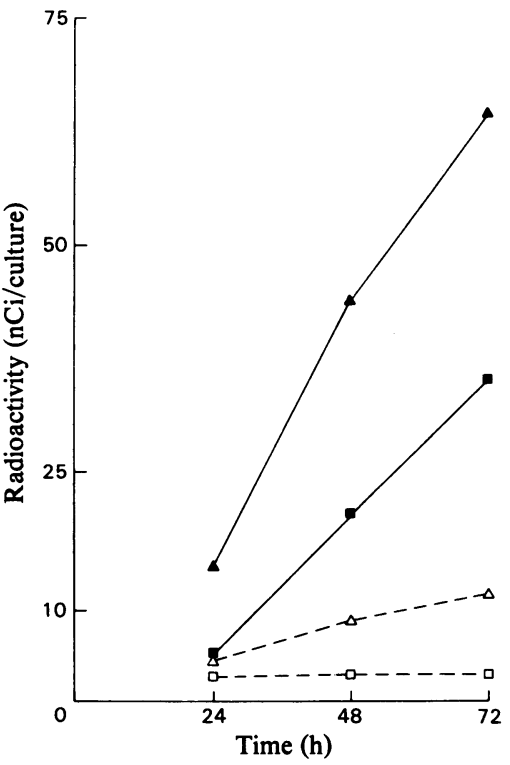

Fig. 1. Polyamine formation by human lymphocytes grown in vitro

Cells were grown in the presence of $\left[{ }^{14} \mathrm{C}\right]$ putrescine with or without purified phytohaemagglutinin. Details of assays are given in the Materials and Methods section. The extracted polyamines were separated by t.l.c. on silica gel in cyclohexane/ethyl acetate $(3: 2, v / v)$. The separation pattern was detected by radioautography. ( $\Delta$ ) Spermidine and (D) spermine in transformed cells; $(\Delta)$ spermidine and $(\square)$ spermine in unstimulated cells. The cellular concentrations of putrescine at 24 and $72 \mathrm{~h}$ were 22 and 303, of spermidine 45 and 3078 and of spermine 72 and $1798 \mathrm{pmol} /$ culture, respectively.

Table 1. Paper chromatography of growth medium derived from lymphocyte cultures in vitro in the presence of $\left[1,4-{ }^{14} \mathrm{C}\right]$ putrescine

The radioactive samples were separated on Whatman no. 3 paper in two separate solvents: (a) propan-2-ol/ $85 \%$ (v/v) formic acid/water (8:1:1, by vol.); $(b)$ propan-1-ol/triethylamine/water (85:3:15, by vol.). Further details and sources of the synthetic markers are given in the Materials and Methods section.

\begin{tabular}{|c|c|c|}
\hline Compounds & Solvent a & Solvent b \\
\hline \multicolumn{3}{|l|}{ Markers } \\
\hline$N^{1}$-Acetylputrescine & 0.77 & 0.59 \\
\hline$N^{1}$-Acetylspermidine & - & 0.45 \\
\hline$N^{8}$-Acetylspermidine & - & 0.68 \\
\hline Putrescine & 0.17 & 0.28 \\
\hline Spermidine & 0.05 & 0.54 \\
\hline Spermine & 0.02 & 0.78 \\
\hline \multicolumn{3}{|l|}{ Unknown } \\
\hline Compound A & 0.77 & 0.54 \\
\hline Compound B & 0.65 & 0.4 \\
\hline
\end{tabular}


compound A migrated with $\boldsymbol{R}_{F} \mathbf{0 . 7 7}$ in solvent a and with $R_{F} 0.54$ in solvent b, like acetylputrescine. Compound B migrated with $R_{F} 0.4$ in solvent b, like an $N^{1}$-acetylspermidine marker. For further identi-

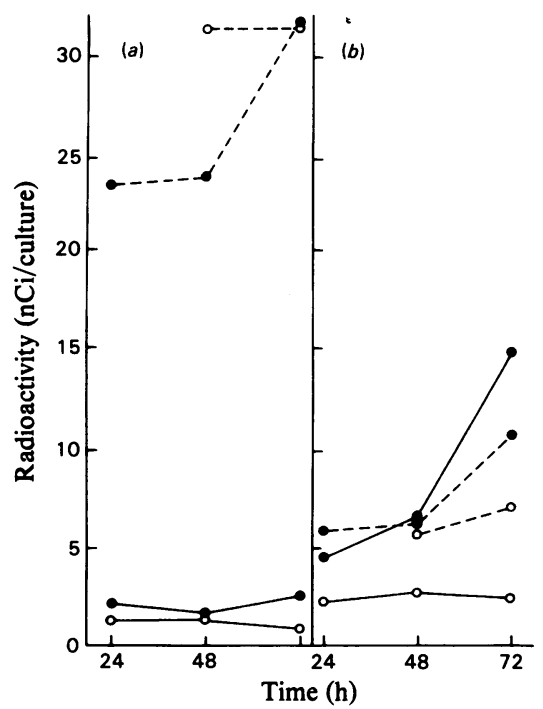

Fig. 2. Formation and release of acetylated polyamines by lymphocytes grown in vitro in the cells and in the growth medium

Cells were grown in the presence of $\left[{ }^{14} \mathrm{C}\right]$ putrescine with or without purified phytohaemagglutinin. The extracted polyamines were separated by t.l.c. in benzene/methanol $(19: 1, \mathrm{v} / \mathrm{v})$ as solvent and detected by radioautography. (a) Acetylputrescine; (b) acetylspermidine. Transformed cells ( and medium (--- $)$; unstimulated cells $(\mathrm{O}-\mathrm{O})$ and medium $(\mathrm{O}---\mathrm{O})$. fication the radioactive spots were eluted from the paper chromatogram, dansylated and separated by two-dimensional t.l.c. Fig. 3(a) illustrates that compound A migrated like acetylputrescine in two different solvents. Fig. 3(b) illustrates that compound B migrated like acetylspermidine. The identity of these two compounds was further confirmed by hydrolysing samples of labelled material eluted from the paper chromatogram. Fig. 4(a) shows that compound A yielded exclusively putrescine. Fig. $4(b)$ demonstrates that compound B yielded mainly spermidine, with traces of spermine.

A final piece of evidence for identification of acetylputrescine was provided by mass spectrometry. The biological compound A yielded peaks at $m / e 363,250,285$ and 169 , like the synthetic dansyl-acetylputrescine.

\section{Discussion}

Human lymphocytes stimulated to growth in vitro seem to be an appropriate model for the study of the metabolic activity of human cells in the resting and the proliferating state (Ling \& Kay, 1975). These cells can be readily obtained and easily stimulated to proliferate. Ultimately, they may serve for the detection of inherited disorders, and thus provide an analytical tool for diagnostic purposes. The use of radioactive precursors such as $\left[{ }^{14} \mathrm{C}\right]$ putrescine, employed in our study, renders the measurement of polyamine metabolism more sensitive, and labelled metabolites may be easily detected and identified.

Data in the present paper clearly demonstrate the occurrence of two labelled acetylated polyamine derivatives, $\quad N$-acetylputrescine and $N^{1}$-acetylspermidine, in lymphocyte cultures. The identity of these two compounds is based on the following

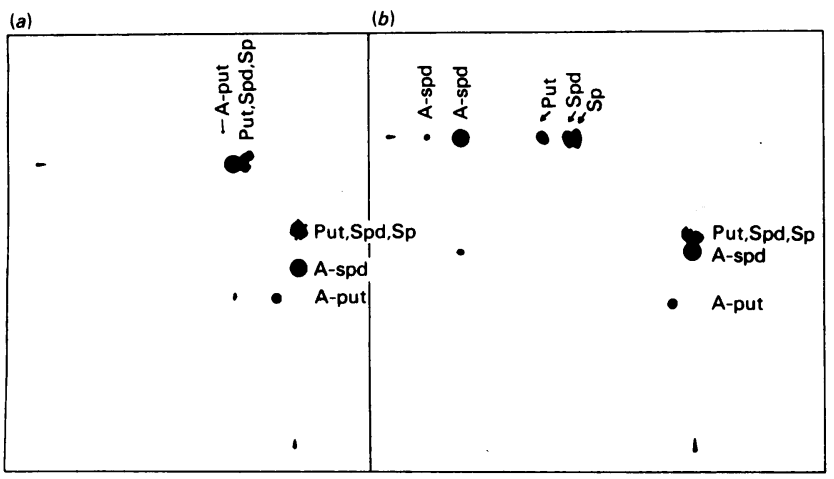

Fig. 3. Two-dimensional thin-layer chromatograms of Compounds $A(a)$ and $B(b)$

The separation pattern was detected by radioautography (Kodak film, Noscreen). The first dimension is from left to right in chloroform/propan-2-ol $(10: 1, \mathrm{v} / \mathrm{v})$ as solvent. The second dimension is upwards in benzene/methanol $(19: 1, \mathrm{v} / \mathrm{v})$ as solvent. Markers: Sp, spermine; Spd, spermidine; Put, putrescine; A-spd, $N^{1}$-and $N^{8}$-acetylspermidine; A-put, acetylputrescine. 

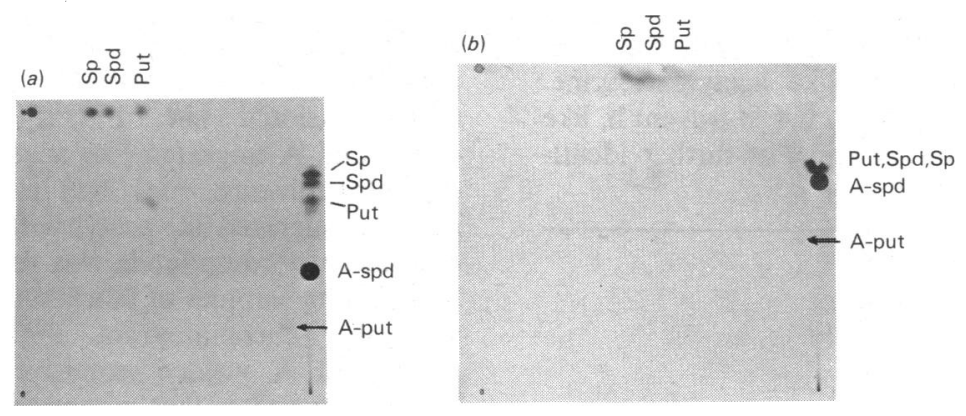

Fig. 4. Two-dimension thin-layer chromatograms of compounds $A(a)$ and $B(b)$ after acid hydrolysis

The material eluted from the paper chromatogram was incubated overnight in $6 \mathrm{M}-\mathrm{HCl}$ at $120^{\circ} \mathrm{C}$. The products of the hydrolysis were dansylated and separated by t.l.c. The first dimension was from left to right in cyclohexane/ethyl acetate $(9: 6, v / v)$ as solvent. The second dimension was in benzene/methanol $(19: 1, v / v)$ as solvent. For key see Fig. 3.

evidence: (a) migration similar to that of synthetic markers on paper chromatography and t.l.c. (Table 1 and Fig. 3); (b) the products obtained after acid hydrolysis of compounds A and B yield putrescine and mainly spermidine respectively (Fig. 4); (c) the mass spectrum of acetylputrescine derived from the lymphocytes.

Acetylation is an attractive mechanism for the physiological inactivation of polyamines. Most of the physiological functions suggested for the polyamines are dependent on the net positive charge carried by these compounds (Cohen, 1971). Acetylation would serve to decrease this net positive charge and thus might modify the physiological activity of polyamines (Blakenship \& Walle, 1978).

Blakenship \& Walle (1978) provided evidence that nuclei and chromatin of rat tissue contain an acetyl-CoA-dependent $N$-acetyltransferase activity which catalyses the $\mathrm{N}$-acetylation of spermidine, spermine and putrescine. Their data also indicate that an enzyme activity is present in a variety of tissues which can metabolize the acetylated polyamines once they are formed. This metabolic process apparently involves deacetylation of the acetylpolyamine. This metabolic activity is detectable in the cytoplasmic fraction of liver and kidney. Seiler \& Al-Therib (1974) found that an oxidative deamination of acetylputrescine occurred in addition to deacetylation.

The appearance of acetylated spermidine in urine (Abdel-Monem et al., 1975) suggests that acetylation may be involved in the excretion of the polyamines. This suggestion is supported by the fact that polyamine acetyltransferase activity is found in the kidney and liver (Libby, 1978). In our experiments, acetylputrescine was detected in the growth medium in relatively high quantities, whereas minute amounts of this compound were retained by the cells. However, acetylspermidine, which is a more positively charged molecule, was not as readily excreted; it was detected in both cells and medium (Fig. 2). The higher concentration of $\mathrm{N}$-acetylspermidine in the stimulated cultures may be related to the increased ability of these cells to synthesize spermidine (Fig. 1). Our studies indicated the occurrence of $N^{1}$-acetylspermidine, but the formation of $N^{8}$-acetylspermidine cannot be excluded. Further studies are warranted to clarify this point.

This study was supported by a grant from Mifal Hapayis to J. F.

\section{References}

Abdel-Monem, M. M., Ohno, K., Fortuny, I. E. \& Theologides, A. (1975) Lancet ii, 1210

Bachrach, U. (1973) Functions of Naturally Occurring Polyamines, pp. 1-211, Academic Press, New York and London

Bachrach, U. (1978) Adv. Polyamine Res. 1, 83-91

Blakenship, J. \& Walle, T. (1978) Adv. Polyamine Res. 2, 97-110

Cohen, S. S. (1971) Introduction to the Polyamines, pp. 1-179, Prentice Hall, Englewood Cliffs, NJ

Dubin, D. T. \& Rosenthal, S. M. (1960) J. Biol. Chem. 235, 783-786

Fillingame, R. H. \& Morris, D. R. (1973) Biochem. Biophys. Res. Commun. 52, 1020-1025

Jänne, J. \& Raina, A. (1968) Acta Chem. Scand. 22, 1349-1351

Kakimoto, Y., Nakajima, T., Kumon, A., Matsuoka, Y., Imaoka, N., Sano, I. \& Kanazawa, A. (1969) J. Biol. Chem. 244, 6003-6007

Kay, J. E. \& Lindsay, V. J. (1973) Exp. Cell Res. 77, $428-436$

Libby, P. R. (1978) Arch. Biochem. Biophys. 188, $360-363$ 
Ling, N. R. \& Kay, J. E. (1975) Lymphocyte Stimulation, pp. 357-375, North-Holland Publishing Co., Amsterdam

Morris, D. R. (1978) Adv. Polyamine Res. 1, 105-115

Noto, T., Tanaka, T. \& Nakajima, T. (1978) J. Biochem. (Tokyo) 83, 543-552

Perry, T. L., Hansen, S. \& MacDougall, L. (1967) J. Neurochem. 14, 775-782

Russell, D. H. \& Durie, B. G. M. (1978) Polyamines as Biochemical Markers of Normal and Malignant Growth, pp. 1-178, Raven Press, New York

Seiler, N. \& Al-Therib, M. J. (1974) Biochim. Biophys. Acta 354, 206-212
Seiler, N. \& Wiechmann, M. (1967) Hoppe-Seyler's Z. Physiol. Chem. 348, 1285-1290

Seiler, N., Schneider, H. \& Sonnenberg, K. D. (1970) Fresenius Z. Anal. Chem. 252, 127-136

Seiler, N., Al-Therib, M. J. \& Knödgen, B. (1973) Hoppe-Seyler's Z. Physiol. Chem. 354, 589-590

Tabor, C. W. \& Tabor, H. (1976) Annu. Rev. Biochem. 45, 285-306

Thorsby, E. \& Bratlie, A. (1970) in Histocompatibility Testing 1970 (Terasaki, P. I., ed.), pp. 655-656, Munksgaard, Copenhagen 